\title{
Combined multivariate statistical and flux balance analyses uncover media bottlenecks to the growth and productivity of $\mathrm{CHO}$ cell cultures
}

\author{
Hock Chuan Yeo ${ }^{1}$, Seo-Young Park ${ }^{2}$, Tessa Tan ${ }^{1}$, Say Kong Ng${ }^{1}$, Meiyappan Lakshmanan ${ }^{1}$, \\ and Dong-Yup Lee ${ }^{2}$ \\ ${ }^{1}$ Bioprocessing Technology Institute \\ ${ }^{2}$ Sungkyunkwan University College of Natural Science
}

July 22, 2021

\begin{abstract}
Chinese hamster ovary $(\mathrm{CHO})$ cells are widely used for producing recombinant proteins. To enhance their growth, productivity, and product quality, practically media reformulation has been one of key focuses with several technical challenges which are due to the myriad of intricate molecular and regulatory mechanisms underlying the media effects on culture behaviours; it is highly required to systematically characterize metabolic bottlenecks of cell cultures in various media conditions. To do so, we combined multivariate statistical analysis with flux balance analysis of a genome-scale metabolic model of CHO cells based on the culture profiles of CHO-DG44 under one commercial medium and two in-house media. At the outset, we used partial least square regression to identify metabolite exchanges that are correlated to specific growth and productivity. By using a commercial medium as reference, we found sub-optimal level of four nutrients and two metabolic wastes that plausibly hinder cell growth and productivity with in-house media. Subsequently, we elucidated that the recycling of lactate and ammonia wastes to be affected by both glutamine and asparagine metabolisms mechanistically, and further modulated by hitherto unsuspected folate and choline supplements. In summary, the current work successfully demonstrated how multivariate statistical analysis can be synergistically combined with in silico analysis of metabolic models to uncover the mechanistic elements underlying the differing performance of various media. Our approach for the systematic identification of promising nutrient targets thus paves the way for cell culture medium reformulation to enhance cellular growth and recombinant protein production.
\end{abstract}

\section{Hosted file}

Media_manuscript_final.doc available at https://authorea.com/users/427004/articles/531320combined-multivariate-statistical-and-flux-balance-analyses-uncover-media-bottlenecksto-the-growth-and-productivity-of-cho-cell-cultures 

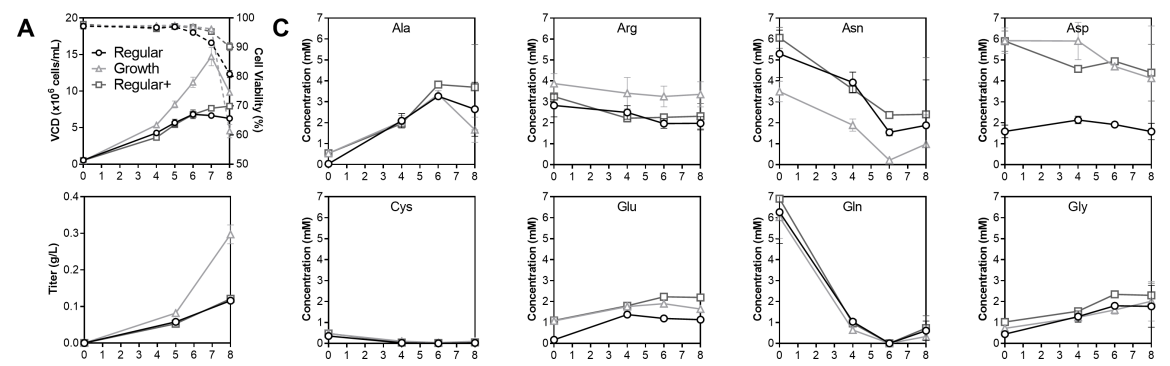

B
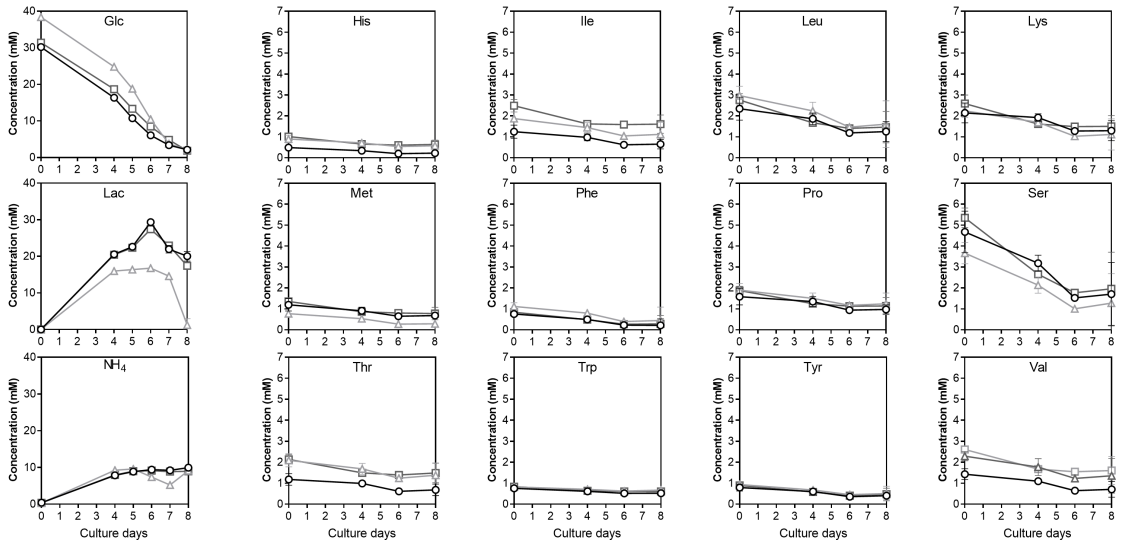

A

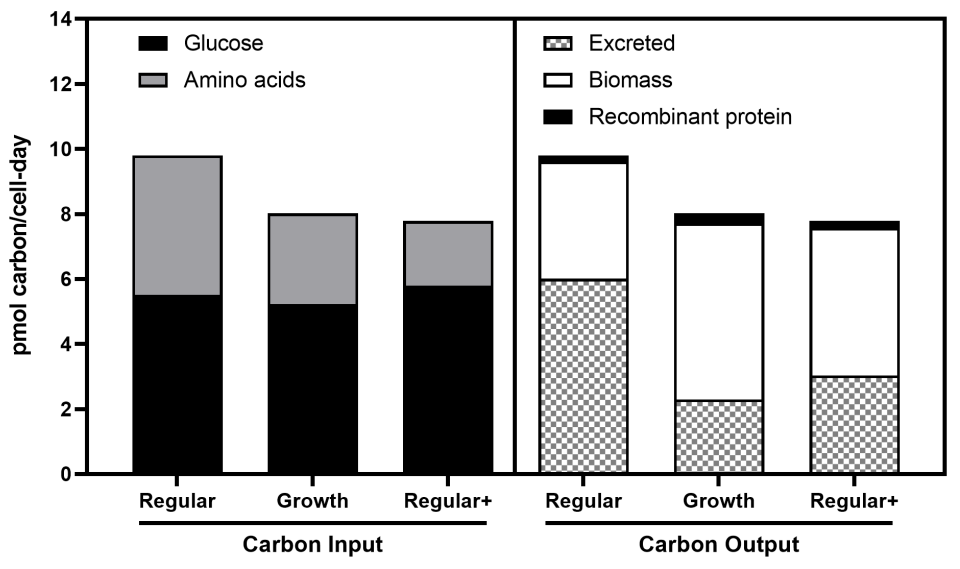

B

Specific growth

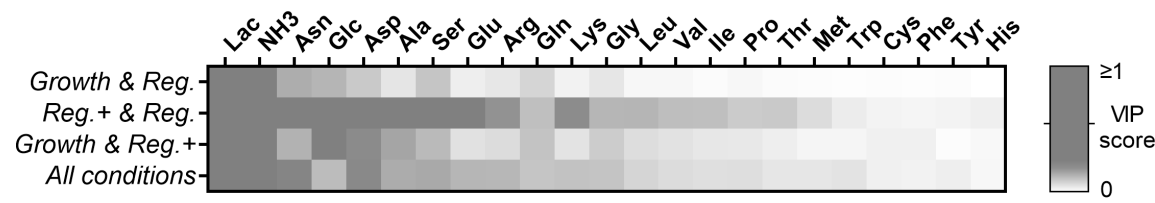

Specific productivity

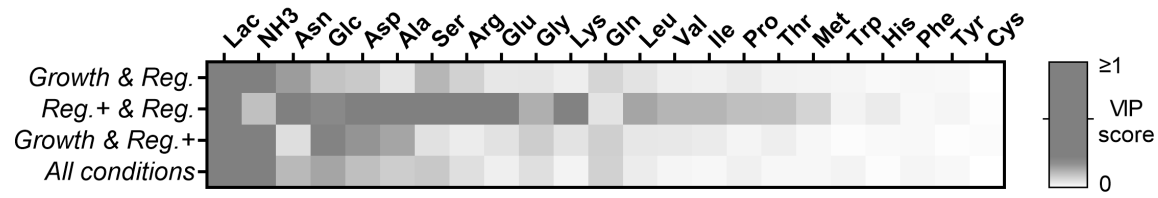


A
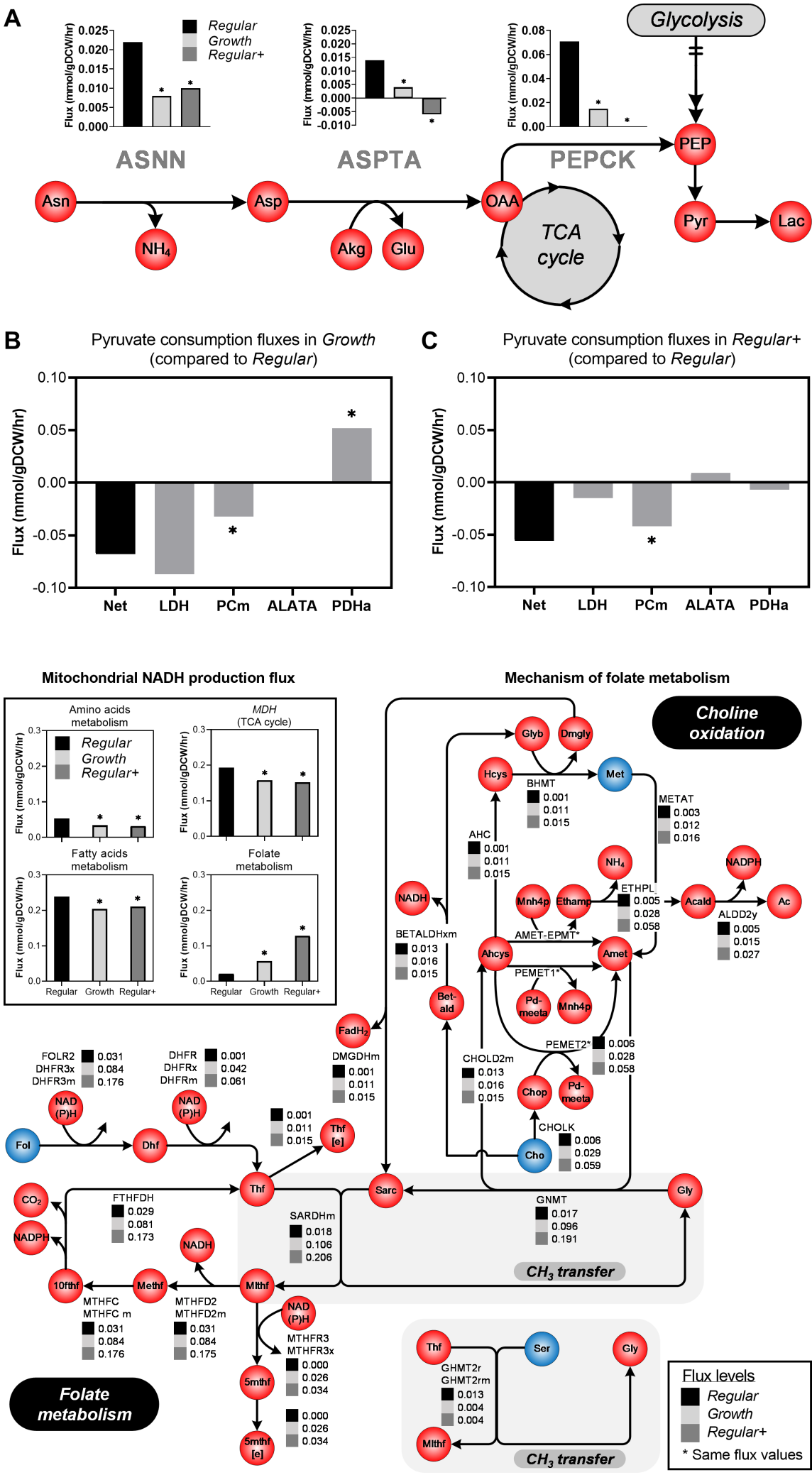
A $\quad \mathrm{NH}_{4}$ production by nutrient source

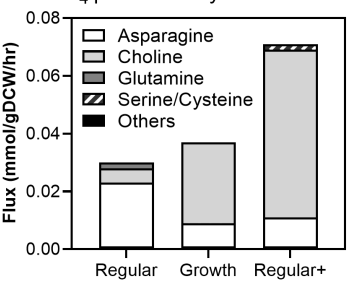

B

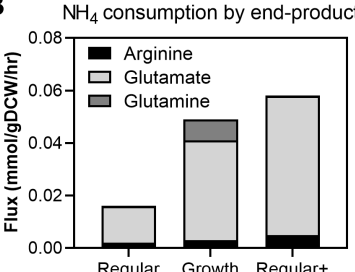

C

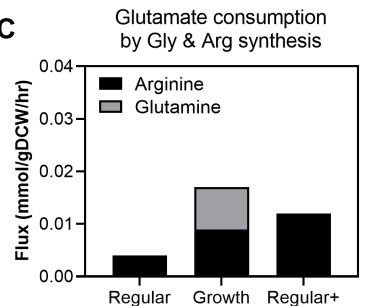

D

Redox effect on Arginine biosynthesis

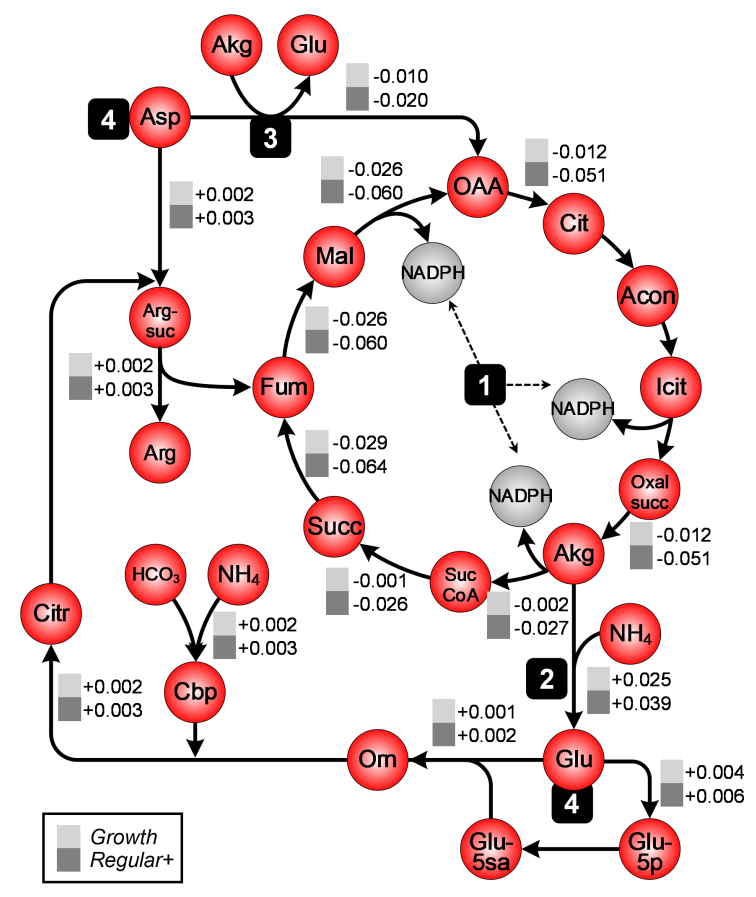




\section{Step 1. Culture profiling in different media}

$\underline{\mathrm{CHO} \text { culture in different media conditions }}$

Culture profiles

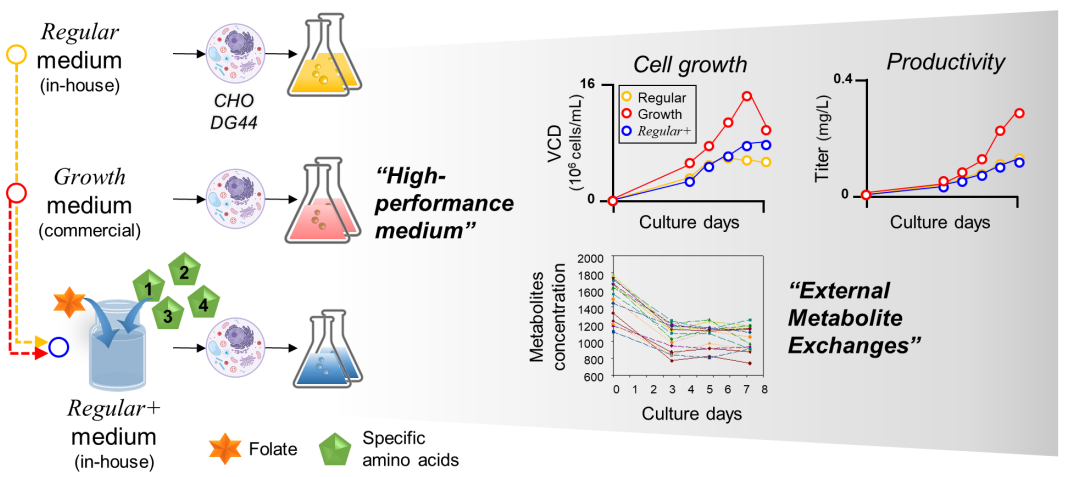

Step 2. Multivariate statistical analysis: Identifying metabolite exchanges correlated with growth \& productivity

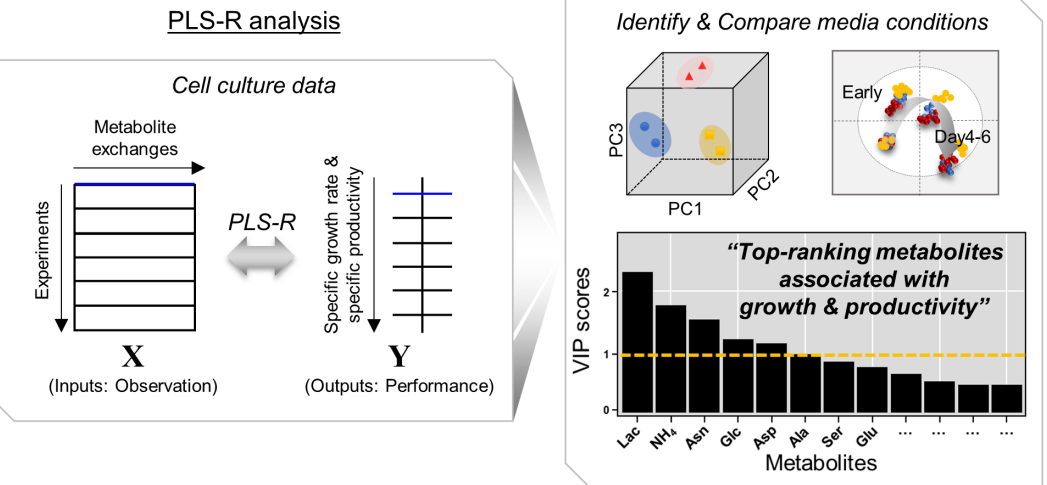

Step 3. In-silico modeling: Understanding mechanisms of action

- Specific exchange rates of metabolites

- Specific growth \& productivity

- Correlated metabolite exchange

$\rightarrow$ Glycolysis \& TCA cycle

$\rightarrow$ Folate \& Choline metabolism

$\rightarrow$ Gln. \& Arg. biosynthesis

$=\ldots$

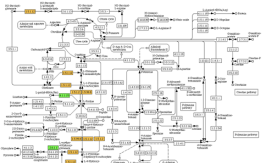

Asn., Lac. \& NH4 exchanges: mechanism of action
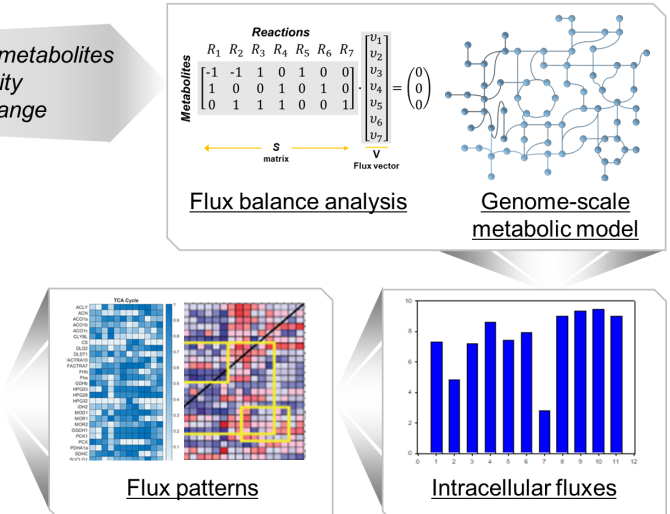